'Departamento de Medicina, Escuela de Medicina, Universidad de Valparaíso. Valparaíso, Chile. ${ }^{2}$ Sección Nefrología, Servicio de Medicina, Hospital Naval A. Nef, Viña del Mar, Chile. ${ }^{3}$ Sección Nefrología, Servicio de Medicina, Hospital Dr. Gustavo Fricke, Viña del Mar, Chile.

Recibido el 14 de septiembre de 2015, aceptado el 14 de diciembre de 2015

Correspondencia a: Dr. Jorge Vega 5 Norte 1035, Viña del Mar, Chile.

Teléfono 56-32-2974237 jvegastieb@gmail.com

\section{Compromiso renal en síndrome de POEMS. Caso clínico}

\author{
JORGE VEGA ${ }^{1,2,3}$
}

\section{Chronic kidney disease associated with POEMS syndrome. Report of one case}

POEMS syndrome is characterized by Polyneuropathy, Organomegaly, Endocrinopathy, Monoclonal protein and Skin changes. We report a woman with the syndrome, who had peripheral polyneuropathy, osteosclerotic myeloma, monoclonal IgA elevation, hypothyroidism, hypogonadotrophic hypogonadism, hyperprolactinemia, adrenal insufficiency, hepatosplenomegaly, lymphadenopathy, thyroid and parotid enlargement, Castleman's disease, papilledema, stiff and hyperpigmented skin, white nails, clubbing, ascites and chronic diarrhea. She had also a nephropathy characterized by microscopic hematuria, proteinuria, renal insufficiency and a unilateral kidney retraction. She was treated with melphalan and prednisone, achieving remission of the disease and nephropathy. She survived twelve years and died due to a myocardial infarction 20 years after POEMS diagnosis.

(Rev Med Chile 2016; 144: 516-520)

Key words: Kidney Failure, Chronic; Multiple Myeloma; Renal Insufficiency, Chronic; Vascular Endothelial Growth Factors.
$\mathrm{E}$ 1 síndrome de POEMS (SP) es una rara manifestación paraneoplásica de un desorden monoclonal de células plasmáticas. POEMS es un acrónimo en inglés de poliradiculoneuropatía, organomegalia, endocrinopatía, desorden clonal de células plasmáticas y alteraciones cutáneas. La mayoría de los casos se han descrito en población asiática, pero hay comunicaciones en occidente y también en Chile ${ }^{1-4}$.

El SP puede comprometer los riñones. La frecuencia del compromiso renal no está precisada $^{1,6,7}$. Puede manifestarse solamente por alteraciones del examen de orina (hematuria y/o proteinuria) o comprometer la función renal severamente, requiriendo diálisis ${ }^{7}$. Las biopsias renales muestran características morfológicas que parecen deberse a la acción de una alta concentración de citoquinas y factores de crecimiento originadas en las células plasmáticas monoclonales ${ }^{1,7}$.

Se comunica el caso de una paciente con SP y afectación renal que tuvo una larga evolución de esta patología. El propósito de la comunicación es el advertir a los lectores de la posibilidad de compromiso renal en esta rara enfermedad.

\section{Caso clínico}

Mujer de 30 años, sin antecedentes mórbidos, a quien a raíz de una consulta por un trastorno en la marcha bilateral ("steppage"), se diagnosticó una neuropatía periférica motora, que se confirmó con medición de conducción nerviosa. Destacaba hiperpigmentación cutánea.

Reapareció 6 años más tarde relatando que había dejado de trabajar por debilidad de extremidades inferiores. La hiperpigmentación había aumentado, su peso disminuido, estaba en amenorrea y sufría diarrea en forma crónica. $\mathrm{Al}$ examen físico destacó una piel dura, acartonada, oscura y descamativa sin hiperpigmentación de pliegues; uñas blancas en vidrio de reloj, bocio 
difuso firme, hipertrofia parotídea, mamas duras sin nódulos, adenopatías axilares bilaterales de $2-3 \mathrm{~cm}$, soplo sistólico en foco pulmonar, palpación abdominal dificultosa por endurecimiento cutáneo y signos claros de neuropatía periférica motora en miembros inferiores. Se plantearon los diagnósticos diferenciales de enfermedad de Addison, esclerodermia, hepatopatía autoinmune y hemocromatosis; los que fueron descartados razonablemente (Tabla 1). El laboratorio reveló un hipotiroidismo con anticuerpos anti-tiroideos ausentes, cortisol basal normal con elevación insuficiente al estímulo con ACTH, hipogonadismo hipogonadotrófico, hiperprolactinemia, hematuria microscópica, proteinuria e insuficiencia renal moderada (Tabla 1). Las imágenes mostraron hepatoesplenomegalia, ascitis, riñones ecogénicos con mala diferenciación corticomedular y un aracnoidocele intraselar. La biopsia hepática fue normal. Se prescribió tiroxina $50 \mathrm{ug} /$ día y cortisol $20 \mathrm{mg} /$ día, con mejoría anímica.

$\mathrm{Al}$ año siguiente presentaba ascitis en tensión con eversión umbilical. El líquido fue un exudado con cultivo corriente y de Koch negativos y ADA normal. Había papiledema bilateral. La proteinuria persistía y la función renal se dete- rioró (Tabla 1). El scanner mostró engrosamiento de piel y tejido celular subcutáneo; adenopatías axilares, mediastínicas y retroperitoneales; hepatoesplenomegalia, signos de nefropatía crónica con reducción de volumen del riñón derecho con escasa eliminación del medio de contraste y una lesión osteoesclerótica en la séptima costilla derecha. Se diagnosticó un SP. Existía un componente monoclonal en la electroforesis de proteínas plasmáticas con una concentración de IgA 4 veces lo normal. El mielograma era inespecífico, la biopsia costal mostró un mieloma osteoesclerótico y la de ganglio linfático una enfermedad de Castleman. Se prescribió 12 ciclos mensuales de melfalan y prednisona.

En los siete años posteriores a la quimioterapia la enferma se controló regularmente. La ascitis no volvió a repetirse, desapareció el componente monoclonal, la lesión costal no mostró variaciones y la función renal y proteinuria se estabilizaron (Tabla 1).

Se perdió de los controles durante 4 1/2 años reapareciendo por una trombosis de arteria iliaca común derecha. Fue revascularizada quirúrgicamente, sufriendo una injuria renal aguda no oligúrica en el postoperatorio. La electroforesis de

Tabla 1. Exámenes bioquímicos y hematológicos durante su evolución

\begin{tabular}{|c|c|c|c|c|c|c|c|c|c|c|}
\hline & $\begin{array}{c}\text { Año } \\
1\end{array}$ & $\begin{array}{c}\text { Año } \\
7\end{array}$ & $\begin{array}{c}\text { Año } \\
8\end{array}$ & $\begin{array}{c}\text { Año } \\
9\end{array}$ & $\begin{array}{c}\text { Año } \\
10\end{array}$ & $\begin{array}{c}\text { Año } \\
11\end{array}$ & $\begin{array}{c}\text { Año } \\
12\end{array}$ & $\begin{array}{c}\text { Año } \\
13\end{array}$ & $\begin{array}{c}\text { Año } \\
14\end{array}$ & $\begin{array}{c}\text { Año } \\
18\end{array}$ \\
\hline Creatinina (mg/dl) $(0,57-1,11)$ & & 2,0 & 3,5 & 2,56 & 2,14 & 2,4 & 2,82 & 2,44 & & \\
\hline Urea (mg/dl) $(14,9-43)$ & 34 & 65 & 137 & 93 & 90 & 69 & 61 & 61 & & \\
\hline Bicarbonato (mEq/L) (20-31) & & 12,9 & 15,3 & & & & & & 16,6 & 18,9 \\
\hline Anion Gap (mEq/L) (8-16) & & 10,3 & 22,8 & & & & & & 22,3 & \\
\hline Proteinuria (g/24 h) $(<0,150)$ & & 1,36 & 1,40 & 1,31 & 0,65 & 0,50 & & 2,42 & 1,51 & \\
\hline $\begin{array}{l}\text { Clearance de creatinina/24 h } \\
\left(\mathrm{ml} / \mathrm{min} / 1,73 \mathrm{~m}^{2}\right)(>90)\end{array}$ & & 41 & 24 & 38,5 & & 52,5 & & 36,0 & & \\
\hline Hematocrito (\%) (37-47) & 40 & 45 & 33,1 & 33,3 & 27,9 & & & 34,8 & 33,9 & 30,5 \\
\hline Hemoglobina (g/dl) (12-16) & & 15,0 & 10,6 & 11,2 & 9,1 & & & 11,7 & 11,6 & 10,0 \\
\hline $\begin{array}{l}\text { Leucocitos }\left(\mathrm{mm}^{3}\right) \\
(4.800-10.800)\end{array}$ & & 5.000 & 5.600 & 5.400 & 3.300 & & & 5.400 & 6.800 & 7.900 \\
\hline Plaquetas $\left(\mathrm{mm}^{3}\right)(140-400)$ & & 382.000 & & 474.000 & 222.000 & & & 340.000 & 316.000 & 318.000 \\
\hline VHS (mm/h) (1-20) & & 24 & 38 & 66 & & & & 63 & 57 & \\
\hline Acido úrico $(\mathrm{mg} / \mathrm{dl})(2,6-6,0)$ & & & 8,9 & 7,8 & 7,7 & 7,5 & 6,8 & 7,3 & & 5,2 \\
\hline $\begin{array}{l}\text { Proteína } C \text { reactiva } \\
\text { (hasta } 0,6)(\mathrm{mg} / \mathrm{L})\end{array}$ & & & 1,16 & & & & & & & 1,29 \\
\hline
\end{tabular}


Tabla 2. Exámenes hormonales e inmunológicos durante su evolución

\begin{tabular}{|c|c|c|c|c|c|c|c|c|c|}
\hline & $\begin{array}{c}\text { Año } \\
1\end{array}$ & $\underset{7}{\text { Año }}$ & $\begin{array}{c}\text { Año } \\
8\end{array}$ & $\begin{array}{c}\text { Año } \\
9\end{array}$ & $\begin{array}{c}\text { Año } \\
10\end{array}$ & $\begin{array}{c}\text { Año } \\
11\end{array}$ & $\begin{array}{c}\text { Año } \\
13\end{array}$ & $\begin{array}{c}\text { Año } \\
14\end{array}$ & $\begin{array}{c}\text { Año } \\
18\end{array}$ \\
\hline \multicolumn{10}{|l|}{ Exámenes inmunológicos } \\
\hline $\lg \mathrm{g}(\mathrm{mg} / \mathrm{dl})(700-1.600 \mathrm{mg} / \mathrm{dl})$ & & & 1.435 & & & 1.421 & 1.649 & 2.127 & 1.530 \\
\hline $\operatorname{lgA}(\mathrm{mg} / \mathrm{dl})(70-140 \mathrm{mg} / \mathrm{dl})$ & & & 637 & & & 538 & 677 & 638 & 575 \\
\hline $\operatorname{lgM}(\mathrm{mg} / \mathrm{dl})(40-230 \mathrm{mg} / \mathrm{dl})$ & & & 100 & & & 264 & 72 & 109 & 60,7 \\
\hline $\mathrm{C}^{\prime} 3 \mathrm{mg} / \mathrm{dl}(70-140 \mathrm{mg} / \mathrm{dl})$ & & & 93 & & & & & & \\
\hline$C^{\prime} 4 \mathrm{mg} / \mathrm{dl}(10-40)$ & & & 13,5 & & & & & & \\
\hline Factor reumatoide & & neg & & & & & & & \\
\hline Anticuerpos antinucleares & & neg & neg & & & & & & \\
\hline Anticuerpos anti-DNA & & & neg & & & & & & \\
\hline Anticuerpos anti-ENA & & & neg & & & & & & \\
\hline ANCA & & & neg & & & & & & \\
\hline Anti-cardiolipinas & & & neg & & & & & & \\
\hline Anti-mitocondriales & & neg & & & & & & & \\
\hline Anti-músculo liso & & & neg & & & & & & \\
\hline Anti-tiroglobulina & & neg & & & & & & & \\
\hline Anti-microsomales & & neg & & & & & & & \\
\hline \multicolumn{10}{|l|}{ Exámenes hormonales } \\
\hline Cortisol plasmático (ug/dl) (5-25) & & 8,5 & & & & & & & \\
\hline Cortisol post ACTH (ug/dl) (> 21) & & 14,9 & & & & & & & \\
\hline $\mathrm{TSH}$ uUI/ml $(0,27-4,2)$ & & 14,09 & & & & & & & \\
\hline T4 $(5,1-13,5)(4,5-12 \mathrm{mcg} / \mathrm{L})$ & & 4,88 & & & & & & & \\
\hline $\mathrm{FSH}(1,55-17,9 \mathrm{mUI} / \mathrm{ml})$ & & 5,9 & & & & & & & \\
\hline LH $(1,31-10,5 \mathrm{mUl} / \mathrm{ml})$ & & 7,1 & & & & & & & \\
\hline PRL (ng/ml) $(2,7-16,9 \mathrm{ng} / \mathrm{ml})$ & & 64 & & & & & & & \\
\hline
\end{tabular}

proteínas plasmáticas no mostró el componente monoclonal. La enferma volvió a ausentarse de los controles, falleciendo de un infarto agudo miocárdico 2 años después, a los 50 años de edad, 20 años después de iniciado el SP, 12 años después de iniciada la quimioterapia y no necesitó diálisis crónica.

\section{Discusión}

Para hacer el diagnóstico de SP se requiere la existencia de 2 criterios obligatorios (polineuropatía y proliferación monoclonal de células plasmáticas), 1 de 3 criterios mayores (enfermedad de Castleman, lesiones osteoescleróticas, elevación del factor de crecimiento vascular endotelial -VEGF) y 1 de 6 criterios menores: 1) organomegalia (esplenomegalia, hepatomegalia, linfoade- nopatías); 2) sobrecarga de volumen extracelular (edema, ascitis, derrame pleural o pericárdico); 3) endocrinopatía (adrenal, tiroidea, pituitaria, gonadal, paratiroidea, pancreática); 4) cambios cutáneos (hiperpigmentación, hipertricosis, hemangiomas glomeruloides, acrocianosis, uñas blancas, bochornos); 5) papiledema; 6) trombocitosis y/o policitemia. También pueden existir otras manifestaciones como uñas en vidrio de reloj, hipertensión pulmonar, patología pulmonar intersticial, diarrea, trombosis y niveles bajos de vitamina $\mathrm{B} 12^{1}$. El diagnóstico en esta enferma cumplió con los 2 criterios obligatorios, 2 de los 3 criterios mayores (no se midió la concentración de VEGF), 5 de los 6 criterios menores y otras manifestaciones.

La frecuencia de compromiso renal en el SP es incierta, aunque parece ser más frecuente que la comunicada en los primeros reportes de este 
trastorno ${ }^{1,6,8-10}$. La mayoría de los pacientes al diagnóstico del SP tiene una creatininemia normal y sólo $6 \%$ tiene $\geq 1,5 \mathrm{mg} / \mathrm{dl}$; sin embargo, si se utiliza la cistatina $\mathrm{C}$ como índice de filtración glomerular, esta frecuentemente está elevada $(71 \%)^{11}$. Otras series han comunicado una frecuencia de creatininemias $>1,5 \mathrm{mg} / \mathrm{dl}$ superior $(50 \%)^{12}$. En una serie china, $37 \%$ de los pacientes tenían un clearance de creatinina $<60 \mathrm{ml} / \mathrm{min} / 1,73 \mathrm{~m}^{2}, 9 \%$ $<30 \mathrm{ml} / \mathrm{min} / 1,73 \mathrm{~m}^{213}$. En otra serie de $299 \mathrm{pa}-$ cientes chinos, $22 \%$ de ellos tenían una velocidad de filtración glomerular $<60 \mathrm{ml} / \mathrm{min} / 1,73 \mathrm{~m}^{27}$. La relación BUN/creatinina a menudo está elevada, sugiriendo contracción de volumen intravascular. Esto es más común en pacientes con ascitis ${ }^{6,12}$.

La mayoría de los pacientes son normoten$\operatorname{sos}^{6,12}$. Las manifestaciones clínicas más frecuentes son la presencia de proteinuria de bajo grado y/o hematuria microscópica. Menos de $10 \%$ de los pacientes tienen proteinuria de $>500 \mathrm{mg} / 24 \mathrm{~h}$ y $15 \%$ tienen hematuria microscópica ${ }^{13}$. La existencia de un síndrome nefrótico o de hematuria macroscópica es excepcional ${ }^{6,12}$. Ocasionalmente se ha comunicado la existencia de acidosis tubular renal tipo $\mathrm{IV}^{9}$.

La necesidad de diálisis más frecuentemente está originada en anasarca intratable que en una falla renal avanzada ${ }^{12}$. Ocasionalmente se ha observado retracción del tamaño de los riñones. Esto puede ocurrir en forma unilateral, como en esta paciente (aunque no pueden en ella excluirse otras causas), o en forma bilateral y se ha atribuido a endarteritis ${ }^{6}$. El intervalo entre el diagnóstico de la enfermedad y la detección de la retracción renal ha variado entre 5 y 12 años ${ }^{12}$.

Entre $11 \%$ y $34 \%$ de los pacientes con SP tienen concomitantemente una enfermedad de Castleman, como esta paciente. La presencia de ambos trastornos aumenta el riesgo de desarrollar una nefropatía ${ }^{1,10}$.

En las biopsias renales de los pacientes con SP se ha encontrado en la microscopía óptica un patrón membranoproliferativo y de daño endotelial tipo microangiopatía trombótica ${ }^{1,14}$. Frecuentemente hay crecimiento glomerular marcado, expansión mesangial por aumento de la matriz y proliferación de células mesangiales y endoteliales, lo que estrecha los lúmenes capilares. También se ha observado engrosamiento de la pared capilar con interposición mesangial circunferencial, microaneurismas y nódulos mesangiales con mesangiolisis ${ }^{7,8,12}$. Puede existir compromiso tubulointersticial inflamatorio o fibrótico de baja cuantía, atrofia tubular, aunque ocasionalmente se ha descrito hiperplasia angiofolicular similar a la enfermedad de Castleman de ganglios linfáticos o infiltración por células plasmáticas ${ }^{6,15-17}$. En las arteriolas puede haber proliferación de la íntima con estenosis severa de sus lúmenes ${ }^{12}$. En un caso se comunicó la presencia de poliarteritis nodosa asociada a un plasmocitoma renal sin compromiso glomerular ${ }^{15}$. La microscopía electrónica habitualmente muestra edema y vacuolización de la célula endotelial, ensanchamiento del espacio subendotelial con la presencia de detritus y duplicación de las membranas basales ${ }^{1,7}$. La presencia de depósitos densos es infrecuente, aunque se ha comunicado la presencia de IgA y cadenas lambda en el espacio subendotelial mediante microscopia inmunoelectrónica ${ }^{18}$. La inmunofluorescencia es habitualmente negativa para inmunoglobulinas y complemento, lo que permite diferenciarlas de las glomerulonefritis membranoproliferativas primarias $^{1,7}$. En esta enferma no se efectuó biopsia renal por tratarse casi de una monorrena funcional.

La patogenia del compromiso renal del SP se desconoce. Se ha postulado que las altas concentraciones de citoquinas y factores de crecimiento como IL- $1 \beta$, IL-6, TNF- $\alpha$ y VEGF producirían daño endotelial a nivel renal y sistémico ${ }^{1,10,19,20}$.

El tratamiento del SP es el propio de las discrasias de células plasmáticas. Se han usado corticoides asociados a drogas alquilantes, trasplante de células troncales autólogas, talidomida, lenalidomida, bortezomib, micofenolato y bevacizu$\mathrm{mab}^{1,6,7,14}$. Estas dos últimas drogas no se emplean en el tratamiento de las discracias monoclonales sino específicamente en el SP, para estabilización de la nefropatía o neuropatía periférica. En esta paciente el empleo de 12 ciclos de melfalan y prednisona logró estabilizar su enfermedad con desaparición de la ascitis y del componente $\mathrm{M}$, sin deterioro posterior de su función renal, permitiéndole sobrevivir 12 años.

El curso clínico del SP es el de una enfermedad crónica con una sobrevida promedio de 14 años. La presencia de uñas en vidrio de reloj, exceso de VEC, hipertensión pulmonar, enfermedad pulmonar intersticial y enfermedad de Castleman tienen una peor sobrevida ${ }^{1}$. Los accidentes vasculares cerebrales, infarto al miocardio, caquexia, infecciones y anasarca suelen ser las causas de 
fallecimiento ${ }^{1}$. Esta paciente en sus años finales sufrió de 2 eventos cardiovasculares, el último de los cuales originó su fallecimiento.

\section{Referencias}

1. Dispenzieri A. POEMS syndrome: 2014 update on diagnosis, risk-stratification, and management. Am J Hematol 2014; 89: 214-23.

2. Chandia M, Salomone C, Bertin P, González S. Síndrome de POEMS con lesión lítica aislada y hemangiomas glomeruloides. Caso clínico. Rev Med Chile 2009; 137: 680-4.

3. Viveros L, Valenzuela MA, Rojas D, Castillo J. Síndrome de POEMS. Caso clínico. Rev Med Chile 1995; 122: 5469.

4. Galleguillos V, Rodríguez MI, Bronfman L, Niklitscheck E, Silva M. Síndrome de POEMS: revisión a propósito de un caso. Rev Med Chile 1991; 118: 789-92.

5. Horta P, Quevedo I. Síndrome de POEMS: Caso clínico. Rev Med Chile 2004; 132: 485-8.

6. Rosser GJ, Reitböck PG, Gray MC, Warnicker P. Successful stabilization of nephropathy in a patient with POEMS (polyneuropathy, organomegaly, endocrinopathy, M-band, skin changes) syndrome on treatment with mycophenolate and steroids: a case report. J Med Case Rep 2010; 4: 63.

7. Ye W, Wang C, Cai QQ, Cai H, Duan MH, Li H, et al. Renal impairment in patients with polyneuropathy, organomegaly, endocrinopathy, monoclonal gammopathy and skin changes syndrome: incidence, treatment and outcome. Nephrol Dial Transplant 2015; 0: 1-9 doi:10.1093/ndt/gfv261

8. Fakatsu A, Tamai H, Nishikawa W, Yoshida F, Takeda A, Kodera K, et al. The kidney disease of Crow-Fukase (POEMS) syndrome: a clinic-pathological study of four cases. Clin Nephrol 1991; 36: 76-82.

9. Soubrier M, Deteix P, Derray G, Dubost JJ, Sauvezie B, Chapman A, et al. Renal manifestation in POEMS syndrome. Nephrologie 1994; 15: 335-8.

10. Ji ZF, Zhang DY, Weng SQ, Shen XZ, Liu HY, Dong L.
POEMS syndrome: a report of 14 cases and review of the literature. ISRN Gastroenterol 2012; 2012: 584287.

11. Stankowski-Drengler T, Gertz MA, Katzmann JA, Lacy MQ, Kumar S, Leung L, et al. Serum immunoglobulin free light chain measurements and heavy chain isotype usage provide insight into disease biology in patients with POEMS syndrome. Am J Hematol 2010; 85: 431-4.

12. Nakamoto Y, Imai H, Yasuda T, Wakui H, Miura AB. A spectrum of clinicopathological features of nephropathy associated with POEMS síndrome. Nephrol Dial Transplant 1999; 14: 2370-8.

13. Dispenzieri A, Kyle RA, Lacy MQ, Rajkumar SV, Therneau TM, Greipp PR, et al. POEMS syndrome: Definitions and long-term outcome. Blood 2003; 101: 2496-506.

14. Sanada S, Ookawara S, Karube H, Shindo T, Goto T, Nakamichi T, et al. Marked recovery of severe renal lesions in POEMS syndrome with high-dose melphalan therapy supported by autologous blood stem cell transplantation. Am J Kidney Dis 2006; 47: 672-9.

15. Jha PK, Vankalakunti M, Bonu R, Babu K, Ballal HS. POEMS syndrome with renal plasmacytoma and classic polyarteritis nodosa: a case report. NDT Plus 2011; 4: 406-9.

16. Machiguchi T, Yoshida H, Tamura T. Macrophage and platelet-infiltrated glomerulonephritis with interstitial angiofollicular hyperplasia in a patient with POEMS syndrome. Nephrol Dial Transplant 2001; 16: 2270-1.

17. El Karoui K, Vuiblet V, Dion D, Izzedine H, Guitard J, Frimat L, et al. Renal involvement in Castleman disease. Nephrol Dial transplant 2011; 26: 599-609.

18. Mizuiri S, Mitsuo K, Sakai K, Hayashi I, Susuki T, Nagai $\mathrm{Y}$, et al. Renal involvement in POEMS syndrome. Nephron 1991; 59: 153-6.

19. Soubrier M, Sauron C, Souweine B, Larroche C, Wechsler B, Guillevin L, et al. Growth factors and proinflammatory cytokines in the renal involvement of POEMS syndrome. Am J Kidney Dis 1999; 34: 633-8.

20. Fukatsu A, Ito Y, Yuzawa Y, Yoshida F, Kato M, Miyakawa $\mathrm{K}$, et al. A case of POEMS syndrome showing elevated serum interleukin 6 and abnormal expression of interleukin 6 in the kidney. Nephron 1992; 62: 47-51. 